\title{
COPD detected with screening: impact on patient management and prognosis
}

\author{
Loes C.M. Bertens ${ }^{1}$, Johannes B. Reitsma', Yvonne van Mourik'1, \\ Jan-Willem J. Lammers ${ }^{2}$, Karel G.M. Moons' ${ }^{1}$, Arno W. Hoes ${ }^{1}$ and \\ Frans H. Rutten ${ }^{1}$
}

\begin{abstract}
Affiliations: 'Julius Center for Health Sciences and Primary care, University Medical Center Utrecht, Utrecht, the Netherlands. ${ }^{2}$ Dept of Pulmonology, Division of Heart and Lungs, University Medical Center Utrecht, Utrecht, the Netherlands.
\end{abstract}

Correspondence: Loes C.M. Bertens, Julius Center for Health Sciences and Primary Care, University Medical Center Utrecht, PO Box 85060, Stratenum 6.131, 3508 AB Utrecht, the Netherlands.

E-mail: L.C.M.Bertens-2aumcutrecht.nl

ABSTRACT It is uncertain whether screening of older persons for chronic obstructive pulmonary disease (COPD) is worthwhile because the effects on patient management and prognosis are unknown.

We aimed to assess the short-term consequences of detecting COPD in frail elderly subjects with dyspnoea, considering pulmonary drug use, hospitalisations and all-cause mortality.

Community-dwelling frail elderly subjects, aged 65 years and older, with dyspnoea, participating in a screening study on COPD and heart failure were included. Final diagnoses were assigned by an expert panel based on all data from the screening strategy, including spirometry. Follow-up data were collected from the general practitioners.

Of the 386 patients, 84 (21.8\%) were received a new diagnosis of COPD. Overall, changes in pulmonary drug prescription during 6 months of follow-up were infrequent $(n=53,13.7 \%$; among new cases of COPD, 15 (17.9\%) out of 84$)$. Of all participants, $25.9 \%$ were hospitalised in the first year of follow-up, with the highest rate in patients with newly detected COPD $(32.1 \%)$.

Many new cases of COPD could be detected by screening frail elderly subjects with dyspnoea, but the impact on patient management seems limited. Our study underlines the importance of obtaining follow-up data to assess the true impact of a (screen-detected) diagnosis of COPD on patient management and outcome.

@ERSpublications

Follow-up data are important when assessing the true impact of a new diagnosis of COPD on management and outcomes http://ow.ly/wVLOP

For editorial comments see page 1397.

Received: Jan 282014 | Accepted after revision: May 012014 | First published online: June 122014

Clinical trial: This study is registered at www.clinicaltrials.gov with identifier number NCT01148719.

Support statement: The study was conducted as part of the Dutch National Care for the Elderly Program (ZonMw-NPO) and supported by a research grant from the Netherlands Organization for Health Research and Development (ZonMw grant 311040302).

Conflict of interest: None declared.

Copyright @ERS 2014 


\section{Introduction}

Chronic obstructive pulmonary disease (COPD) is a leading cause of mortality and morbidity worldwide, and it results in a substantial and growing social and economic burden [1]. The diagnosis of COPD is based on evidence of pulmonary obstruction, preferably in combination with signs and symptoms suggestive of COPD, and with a history of smoking $[1,2]$. As a simultaneous but not always linear decrease in forced vital capacity (FVC) and forced expiratory volume in $1 \mathrm{~s}$ (FEV1) is part of natural ageing, it is more difficult to define a decline in lung function as abnormal in the elderly [3, 4]. Importantly, from a pathophysiological point of view, COPD can at least partly be considered as accelerated ageing of the lungs, and thus its prevalence increases with age [5].

Controversy exists about whether screening for COPD in older adults is useful. Several authors suggest it is not beneficial to screen for COPD in the elderly population at large, and also not in selected patient groups such as smokers or patients with complaints of cough [6-9]. However, current guidelines advocate that physicians should pay special attention to the fact that older people do not always report their respiratory complaints and that, in general, spirometry should be considered more often [2]. Moreover, underdiagnosis of COPD, especially in community-dwelling older primary care patients, has been frequently reported [10-12].

The vigour of the discussion of the potential benefits and harms of screening for COPD is fuelled by the lack of evidence on the actual impact of detecting COPD with screening on patient management, and on the prognosis of newly cases detected in this way. We used data from a diagnostic screening trial to compare the differences in diagnostic yield between the screening strategy and care as usual [13]. Additionally, we investigated whether screen-detected COPD resulted in a change in patient management, and short-term prognosis in terms of hospitalisations, exacerbations, pneumonia and all-cause mortality.

\section{Methods}

\section{Subjects and study design}

The study population was derived from a cluster-randomised trial in community-dwelling frail elderly subjects aged 65 years and over with dyspnoea and/or reduced exercise tolerance, registered in primary care. Patients known to have an established diagnosis of both COPD and heart failure were excluded. Because we were interested in both heart failure and COPD, patients with one of these diagnoses remained eligible, as the other condition could be detected with the screening strategy (i.e. screen-detected COPD in patients with known heart failure and vice versa). Randomisation into the screening-strategy or care-as-usual groups was on a practice level (cluster randomisation). Details on the population and study characteristics were published previously [13]. The study complied with the Declaration of Helsinki and the Medical Ethical Committee of the University Medical Centre Utrecht (Utrecht, the Netherlands) approved the study protocol. This study is registered at www.ClinicalTrials.gov with identifier number NCT01148719. All participants gave written informed consent.

\section{Screening arm}

In short, all participants in this study arm underwent a screening strategy, including history taking, physical examination, ECG, pre- and post-bronchodilator spirometry, blood tests, and echocardiography. History taking included a standardised questionnaire on symptoms, medical history, smoking history and current drug use. Physical examination was performed by an experienced physician in a standardised manner. Spirometric measurements were performed while patients were in a clinically stable condition with a SpiroPerfect spirometer (Welch Allyn, Leiden, the Netherlands). FVC and FEV1 were measured before and $30 \mathrm{~min}$ after administration of ipratropium bromide by inhalation. All measurements were performed in a near-home setting at the general practitioner's (GP's) office by trained technicians from the regional GPs' laboratory (Saltro; Utrecht).

\section{Care-as-usual arm}

For each patient in the care-as-usual arm, complaints of dyspnoea and exercise tolerance were communicated to the treating physician. Further management of these patients was at the discretion of the physician.

\section{Panel diagnosis of COPD}

All participants of the screening arm were evaluated by a consensus panel that decided after plenary discussion whether a diagnosis of COPD and/or heart failure was present, possible or absent. The panel consisted of a GP (F.H. Rutten), a pulmonologist (alternating between J-W.J. Lammers and H.J. Huidekoper; University Medical Center Utrecht, Utrecht, the Netherlands) and a cardiologist (alternating between M.J.M. Cramer, M.A. Nugroho Soenardi and C.G.K.M. Fauser; University Medical Center Utrecht). A diagnosis of COPD was assigned after plenary discussion based on all available data from the 
screening strategy, including an extensive smoking history and self-reported information on inhalation allergy or bronchial hyperresponsiveness. The pre- and post-bronchodilator FEV1 expressed as a percentage of the predicted value according to age, sex and height, the FEV1/FVC ratio, the shape of the expiration curve, and presence or absence of reversibility were considered by the panel [14]. Disease severity was staged according to the Global Initiative for Chronic Obstructive Lung Disease (GOLD) criteria [15]. In all cases, agreement about the diagnoses could be reached after discussion. Patients with a "possible" diagnosis of COPD according to the expert panel were considered to have no COPD in the current analysis.

\section{Follow-up data}

Follow-up data were collected by scrutinising the electronic medical files of the GPs of the participants for both study arms. Data on drug use and smoking cessation were collected 6 months after the screening (screening arm) or inclusion in the study (care-as-usual). Information on hospitalisations, episodes of pneumonia or exacerbations of COPD, and mortality was collected both 6 and 12 months after the screening. An episode of pneumonia was considered to have occurred when the patient was diagnosed as such by the GP and received a prescription of antibiotics, or had a hospital admission with pneumonia as the discharge diagnosis. Exacerbations of COPD were defined as symptomatic deterioration requiring pulsed oral corticosteroids or hospitalisation.

\section{Data analysis}

Characteristics of the participants in the screening arm were tabulated against the COPD status defined as "no COPD", "new COPD", "confirmed COPD" or "former COPD". Patients with the label "no COPD" did not have a prior diagnosis of COPD and had no COPD according to the panel. Patients with new COPD had no prior diagnosis of COPD but received such a diagnosis by the panel. The group "confirmed COPD" were patients known with COPD that was confirmed by the panel. In patients with former COPD, the diagnosis could not be confirmed with spirometry and assessment by the panel, and these patients had other causes for their symptoms including (persistent) asthma. Patients in the care-as-usual arm were classified as having "no COPD", "known COPD" or "new COPD". Two patients, both without COPD, were excluded from the analyses because of withdrawal of their informed consent within a week of the start of the study. There were very few missing values in the dataset $(0.4 \%$ overall). Missing panel diagnoses (COPD $(n=3)$, asthma $(n=1)$ and heart failure $(n=10))$ were not imputed. All other missing values were imputed using multiple imputation techniques [16] except for missing values of the spirometric post-bronchodilator test $(n=6)$, for which the pre-bronchodilator test values were used. All analyses were performed using SPSS 20.0 (SPSS Inc., Chicago, IL, USA).

\section{Results}

Of the 389 participants in the screening arm, 66 (17.0\%) patients were known to have a prior diagnosis of COPD. Three patients could not be evaluated because the spirometry was not interpretable. Of the remaining 386 patients, $236(61.1 \%)$ patients were classified as having no COPD, 84 (21.8\%) with new COPD, $50(13.0 \%)$ with confirmed COPD and $16(4.1 \%)$ patients with former COPD. Baseline characteristics are displayed in table 1 . Of the patients with no COPD, $26(11.0 \%)$ could be classified as having pulmonary restriction (normal FEV1/FVC ratio with a FEV1 or FVC $<80 \%$ predicted). These patients were all overweight (mean $\pm \mathrm{SD}$ body mass index $28.1 \pm 4.5 \mathrm{~kg} \cdot \mathrm{m}^{-2}$ ) and $15(57.7 \%) \mathrm{had}$ concomitant heart failure. Using the newly proposed GOLD criteria [1] for severity grading, $90.3 \%$ of patients (121 out of 134) could be graded as having "more complaints" (Medical Research Council dyspnoea score $\geqslant 2$ ) (table 2).

Changes in prescription of pulmonary drugs 6 months after the assessment are presented in table 3. The total number of patients with no COPD using pulmonary drugs decreased from 41 (17.4\%) at baseline to 31 (13.1\%) after 6 months. Of the patients who continued to take their pulmonary drugs, four had asthma and five bronchial hyperresponsiveness. Pulmonary drug use increased in patients with new COPD from 24 $(28.6 \%)$ at baseline to $32(38.1 \%)$ patients after 6 months. Only one patient stopped pulmonary medication in the group with former COPD. In this group, eight patients had asthma and one had pulmonary hyperresponsiveness, and they all continued their pulmonary medication. In the group of patients with confirmed COPD, pulmonary drug use remained similar: $40(80.0 \%)$ at baseline and $39(78.0 \%)$ patients after 6 months. Six (16.2\%) of the 34 smokers among the participants quit smoking within 6 months but none of the smokers with newly detected COPD quit smoking.

Hospitalisations and mortality rates after 12 months of follow-up are summarised in table 4 . Mortality rates did not differ between patients with newly diagnosed COPD and those with no COPD, but seemed lower than in patients with a former or confirmed COPD. Patients with a new diagnosis of COPD were more often 
TABLE 1 Baseline characteristics of the 386 frail elderly stratified by chronic obstructive pulmonary disease (COPD) status after screening

No COPD New COPD $\quad$ Confirmed COPD $\quad$ Former COPD



TABLE 2 Disease classification according to the Global Initiative for Chronic Obstructive Lung Disease (GOLD) criteria of 2007 and 2013 of patients with new or confirmed chronic obstructive pulmonary disease (COPD)

\section{New COPD}

Confirmed COPD

\begin{tabular}{|c|c|c|}
\hline \multicolumn{3}{|l|}{ GOLD criteria 2007} \\
\hline GOLD I: mild & $1(1.2)$ & $0(0.0)$ \\
\hline GOLD II: moderate & $77(91.6)$ & $40(80.0)$ \\
\hline GOLD III: severe & $5(6.0)$ & $9(18.0)$ \\
\hline GOLD IV: very severe & $1(1.2)$ & $1(2.0)$ \\
\hline \multicolumn{3}{|l|}{ GOLD criteria 2013} \\
\hline A: low risk, fewer symptoms & $9(10.7)$ & $4(8.0)$ \\
\hline B: low risk, more symptoms & $69(82.1)$ & $36(72.0)$ \\
\hline C: high risk, fewer symptoms & $0(0.0)$ & $0(0.0)$ \\
\hline D: high risk, more symptoms & $6(7.2)$ & $10(20.0)$ \\
\hline
\end{tabular}

Data are presented as $\mathrm{n}(\%)$. New COPD: no diagnosis of COPD but COPD according to panel; confirmed COPD: diagnosis of COPD and COPD according to panel. GOLD criteria 2007 [15]: severity grading of COPD based on airflow limitation only: GOLD I, forced expiratory volume in $1 \mathrm{~s}$ (FEV1) $>80 \%$ predicted; GOLD II, FEV $1<50 \%$ to $\leqslant 80 \%$ predicted; GOLD III, FEV $1<30 \%$ to $\leqslant 50 \%$ predicted; GOLD IV, FEV $1 \leqslant 30 \%$ predicted. GOLD criteria 2013 [1]: severity grading of COPD based on symptoms (assessed with Medical Research Council (MRC) dyspnoea scale) and airflow limitation: low risk, GOLD I-II; high risk, GOLD III-IV; fewer symptoms, MRC dyspnoea score 0-1; more symptoms: MRC dyspnoea score $\geqslant 2$. 
TABLE 3 Use of pulmonary drugs of 386 frail elderly subjects at baseline and after 6 months of follow-up, stratified by chronic obstructive pulmonary disease (COPD) status

\begin{tabular}{|c|c|c|c|c|}
\hline & No COPD & New COPD & Confirmed COPD & Former COPD \\
\hline Subjects $n$ & 236 & 84 & 50 & 16 \\
\hline \multicolumn{5}{|l|}{ Baseline } \\
\hline Any pulmonary medication & $41(17.4)$ & $24(28.6)$ & $40(80.0)$ & 13 (81.3) \\
\hline Inhaled medication & $24(10.2)$ & $21(25.0)$ & $39(78.0)$ & 13 (81.3) \\
\hline$\beta$-agonists & $6(2.5)$ & 7 (8.3) & $8(16)$ & $5(31.1)$ \\
\hline Anticholinergics & $11(4.6)$ & $5(6.0)$ & $30(60.0)$ & $7(37.8)$ \\
\hline Other pulmonary medication ${ }^{\#}$ & $18(7.6)$ & $3(3.6)$ & $7(14.0)$ & 2 (12.5) \\
\hline \multicolumn{5}{|l|}{ After 6 months of follow-up } \\
\hline Any pulmonary medication & $31(13.1)$ & $32(38.1)$ & $39(78.0)$ & $12(75.0)$ \\
\hline Inhaled medication & $23(9.8)$ & $30(35.7)$ & 39 (78.0) & $12(75.0)$ \\
\hline$\beta$-agonists & $6(2.5)$ & $5(6.0)$ & $14(28.0)$ & $4(25.0)$ \\
\hline Anticholinergics & $9(3.8)$ & $11(13.1)$ & $30(60.0)$ & 7 (37.8) \\
\hline
\end{tabular}

Data are presented as $n(\%)$ unless otherwise stated. No COPD: no diagnosis of COPD and no COPD according to panel; new COPD: no diagnosis of COPD but COPD according to panel; confirmed COPD: diagnosis of COPD and COPD according to panel; former COPD: diagnosis of COPD but no COPD according to panel. " : oral corticosteroids (70\%), mucolytics (16\%), theophylline $(7 \%)$ and antileukotrienes $(7 \%)$.

admitted to hospital than the other patients in the study (fig. 1). Episodes of pneumonia and/or exacerbations were most frequent in patients with confirmed COPD, followed by patients with former COPD.

Care-as-usual arm

Of the 443 patients in the care-as-usual group, 66 (14.9\%) patients already had a diagnosis of COPD at baseline and $13(2.9 \%)$ patients were newly diagnosed with COPD. Pulmonary drug prescription did not differ between baseline and after 6 months, except for the patients with new COPD, where the prescription increased from six patients at baseline to nine patients after 6 months. Mortality rates and hospitalisations after 12 months of follow-up did not differ from those in the screening arm. Compared with the screening arm, fewer patients experienced an episode of pneumonia and/or exacerbation (29 (6.5\%) versus 46 (11.9\%)

TABLE 4 Mortality, hospitalisations, and episodes of pneumonia or chronic obstructive pulmonary disease (COPD) exacerbations in 386 frail elderly subjects after 6 and 12 months of follow-up, stratified by COPD status

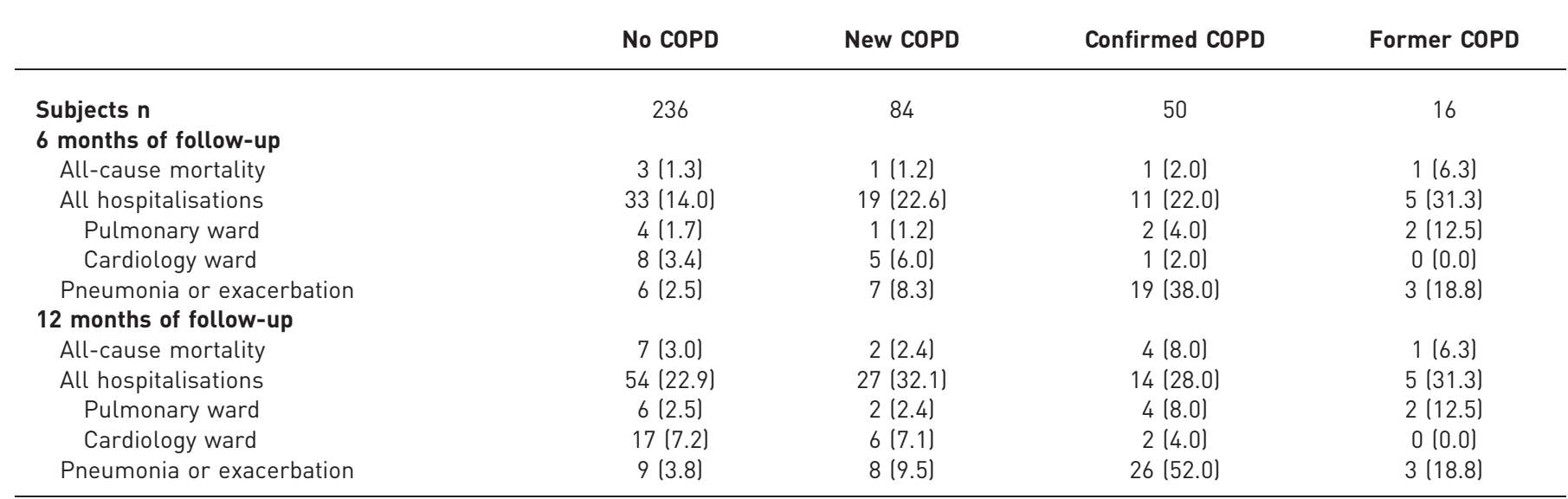

Data are presented as $\mathrm{n}(\%)$ unless otherwise stated. No COPD: no diagnosis of COPD and no COPD according to panel; new COPD: no diagnosis of COPD but COPD according to panel; confirmed COPD: diagnosis of COPD and COPD according to panel; former COPD: diagnosis of COPD but no COPD according to panel. 


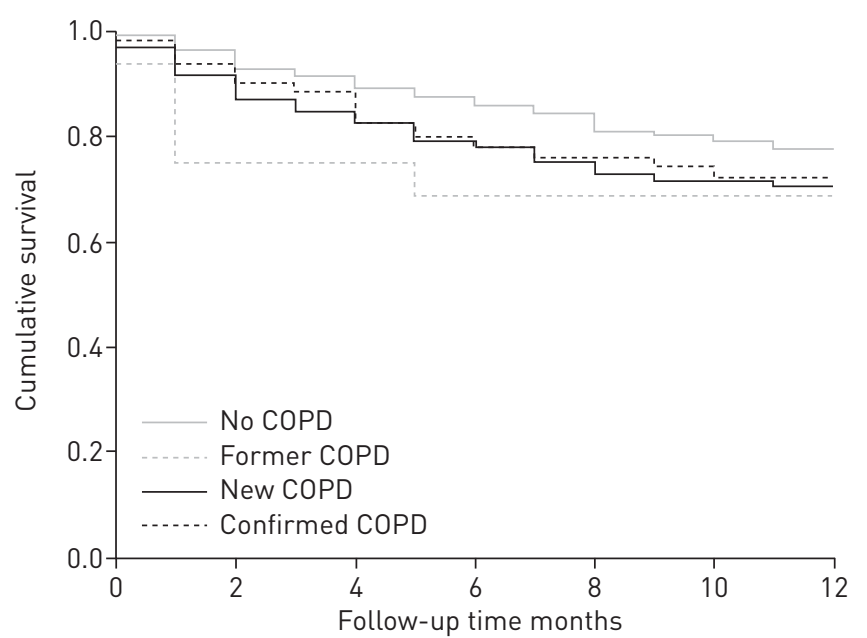

FIGURE 1 Time to first hospital admission stratified by chronic obstructive pulmonary disease (COPD) status. KaplanMeier curve of time (months) to first hospital admission in the screening arm of the study. No COPD: no diagnosis of COPD and no COPD according to panel; new COPD: no diagnosis of COPD but COPD according to panel; confirmed COPD: diagnosis of COPD and COPD according to panel; former COPD: diagnosis of COPD but no COPD according to panel.

in the screening arm), although this was less pronounced in patients with a new diagnosis of COPD (one (7.8\%) versus eight $(9.5 \%)$, respectively).

\section{Discussion}

In our screening study, among frail elderly subjects with complaints of dyspnoea or with exercise intolerance, newly diagnosed COPD was detected in $22 \%$ of the patients. In contrast, a new diagnosis of COPD was found in only 3\% in the care-as-usual arm of the same study. We observed only small changes in pulmonary drug prescription and none of the newly detected COPD patients quit smoking during the 6-month follow-up period. Screen-detected patients had more hospitalisations and exacerbations/pneumonia than elderly subjects without COPD, but similar mortality rates.

Some argue that screening for COPD would only result in the detection of cases with mild disease, which are often not considered clinically relevant. Especially in elderly patients, a decline in pulmonary function is part of the natural ageing process and differentiating between a normal of an abnormal decline becomes difficult. In our study, the majority of patients with newly detected COPD had mild-to-moderate disease based on airflow limitation; however, $90.3 \%$ could be classified as having "more complaints" according to the updated GOLD criteria [1]. This does suggest that screening of frail elderly subjects with shortness of breath results in the detection of clinically relevant COPD.

Several authors have suggested the existence of a "frequent exacerbator phenotype" among COPD patients $[17,18]$. Consequently, a phenotype of COPD patients representing the opposite also exists (infrequent exacerbator). Patients with this latter phenotype are less likely to consult their physician for (an increase in) their complaints of dyspnoea. As a result, they are probably less likely to be invited for a lung function test and considered to have COPD. This hypothesis suggests that the majority of patients with screen-detected COPD in our study represent this infrequent exacerbator phenotype, and the relatively low incidence of episodes of pneumonia and/or exacerbations during follow-up in our study supports this assumption.

There may be multiple reasons why, in the screen-detected COPD patients, patient management hardly ever changed. First of all, older age and fragility may urge doctors to refrain from changing pharmacotherapy because of fear of interfering with a delicate balance and the risk of a cascade of adverse effects after changes in patient management. Second, screen-detected patients possibly profit less from drugs than patients who actively seek contact with a physician because of newly experienced or worsening of symptoms. Consultation because of symptoms can be an important motivator for both patient and physician to change therapeutic management or lifestyle. Third, we did not actively promote changes in patient management. The participating GPs received a diagnosis and patient-tailored treatment advice for each patient directly after the screening strategy, but the GPs had no special training in how subsequently to handle this and received no monitoring data on the changes in drug treatment as a feedback. 
In addition, among patients with no COPD or with former COPD who were already taking pulmonary drugs at baseline, only a few stopped taking the drugs. Apparently, many of these patients experience complaints of dyspnoea, and combined with a tendency of some physicians to treat symptoms, they received pulmonary drugs for symptom relief even without an objective diagnosis of COPD. In addition, the prevalence of asthma $(n=12)$ or pulmonary hyperresponsiveness $(n=6)$ may also have contributed to the decision not to change the prescription of pulmonary medication.

\section{Limitations}

The findings from our study are limited by the included population, i.e. frail elderly persons with complaints of dyspnoea, and may not apply to a younger population, a non-frail population or a population not reporting complaints of dyspnoea.

We only collected data on initiation and discontinuation of pulmonary drug prescriptions. We did not account for changes in the dosage of pulmonary drugs, and could therefore have missed up- and downtitration that could also be considered a relevant change in patient management. We therefore might have underestimated the proportion of patients in whom the management changed after screening. However, pulmonary drug prescription in the patients with screen-detected COPD increased with 10\%. Considering the short time period in which this occurred, and that the treatment intervention of our study was limited to only communicating the novel, screen-detected diagnosis to the GP, this change in prescription could also be considered notable.

We excluded patients known to have a dual established diagnosis of COPD and heart failure before the start of the study and, therefore, the prevalence of COPD in the entire population of frail elderly is higher (44.8\%, 194 out of 433 subjects) than presented by us $(38.6 \%)$. This, however, this does not affect our conclusions regarding newly detected COPD.

We used the electronic medical files of the GPs to collect our follow-up data. Mortality, hospital admissions and data on drug use are known to be accurately recorded in these files [19]. Only moderate-to-severe exacerbations of COPD are included, i.e. those requiring physician attendance, and a short course of oral corticosteroids (with or without antibiotics) or hospitalisation [20].

Some patients could be classified as having pulmonary restriction, using the definition of a normal FEV1/ FVC ratio in combination with a FEV1 or FVC $<80 \%$ predicted. Importantly, the pulmonary restriction as measured with spirometry in our study could be partly explained by overweight and heart failure [21-26].

Finally, some overestimation of newly detected COPD could have occurred because patients with heart failure could be misclassified as COPD due to a reduction in pulmonary function caused by this disease [23]. However, the expert panel consisted of both a pulmonologist and a cardiologist, and both COPD and heart failure were considered simultaneously and extensively, thereby minimising misclassification. Moreover, more extensive pulmonary function testing, including plethysmographic measurements and the use of the residual volume/total lung capacity ratio, are helpful in discriminating between heart failure and COPD, especially in patients with a FEV1/FVC ratio around 0.70 , but these measurements were not available in our study.

\section{Implications for future research}

In the evaluation of diagnostic screening strategies in general, many studies only focus on the diagnostic yield of the screening, without considering the effects further downstream with adjustment of treatment and other types of management. Also, the impact of the strategy on patient-related outcomes is then based on combining the diagnostic yield with estimates of the effectiveness of treatments extrapolated from published randomised controlled trials (RCTs). With such an extrapolation, it is assumed that each patient with a diagnosis will receive treatment, and that the treatment effectiveness in screen-detected cases is similar to the effectiveness demonstrated in these RCTs. However, we have shown in our study among frail elderly subjects that, in real-life clinical practice, not all patients with newly diagnosed COPD will be treated accordingly, especially when detected with screening. It underlines the importance of obtaining follow-up data in screening studies to assess the real-life change in subsequent patient management to evaluate adequately the potential effects of the screening strategy.

\section{Conclusions}

With an active search for COPD in community-dwelling frail elderly subjects with dyspnoea or reduced exercise tolerance, substantially more new patients with COPD can be detected than with care as usual (spirometry in suspected cases). However, the impact on subsequent changes in patient management may be limited, even in patients with a newly established diagnosis. These patients probably benefit most from treatment, as they seem to have a worse prognosis than those without COPD. Our study illustrates the 
importance of carefully collecting follow-up data and the comparison to current practice in screening studies to assess the true impact of the strategy on patient management.

\section{Acknowledgements}

We thank the participating patients, GPs, their assistants and the personnel from Saltro laboratory (Utrecht, the Netherlands). We thank M.A. Nugroho Soenardi (cardiologist), C.G.K.M. Fauser (cardiologist), M.J. Landman (cardiologist), M.J.M. Cramer (cardiologist) and H.J. Huidekoper (pulmonologist) for their contribution to the expert panel (all University Medical Center Utrecht, Utrecht, the Netherlands).

\section{References}

1 Vestbo J, Hurd SS, Agusti AG, et al. Global Strategy for the Diagnosis, Management, and Prevention of Chronic Obstructive Pulmonary Disease: GOLD Executive Summary. Am J Respir Crit Care Med 2013; 187: 347-365.

2 Qaseem A, Wilt TJ, Weinberger SE, et al. Diagnosis and management of stable chronic obstructive pulmonary disease: a clinical practice guideline update from the American College of Physicians, American College of Chest Physicians, American Thoracic Society, and European Respiratory Society. Ann Intern Med 2011; 155: 179-191.

3 Bhatt NY, Wood KL. What defines abnormal lung function in older adults with chronic obstructive pulmonary disease? Drugs Aging 2008; 25: 717-728.

4 Sorino C, Battaglia S, Scichilone N, et al. Diagnosis of airway obstruction in the elderly: contribution of the SARA study. Int J Chron Obstruct Pulmon Dis 2012; 7: 389-395.

Ito K, Barnes PJ. COPD as a disease of accelerated lung aging. Chest 2009; 135: 173-180.

Culver BH. Interpretation of spirometry: we can do better than the GOLD standard. Respir Care 2006; 51: 719-721. Hardie JA, Buist AS, Vollmer WM, et al. Risk of over-diagnosis of COPD in asymptomatic elderly never-smokers. Eur Respir J 2002; 20: 1117-1122.

Schermer TR, Quanjer PH. COPD screening in primary care: who is sick? Prim Care Respir J 2007; 16: 49-53. Swanney MP, Ruppel G, Enright PL, et al. Using the lower limit of normal for the FEV1/FVC ratio reduces the misclassification of airway obstruction. Thorax 2008; 63: 1046-1051.

10 Bednarek M, Maciejewski J, Wozniak M, et al. Prevalence, severity and underdiagnosis of COPD in the primary care setting. Thorax 2008; 63: 402-407.

11 Geijer RM, Sachs AP, Hoes AW, et al. Prevalence of undetected persistent airflow obstruction in male smokers 40-65 years old. Fam Pract 2005; 22: 485-489.

12 Broekhuizen BD, Sachs AP, Hoes AW, et al. Undetected chronic obstructive pulmonary disease and asthma in people over 50 years with persistent cough. Br J Gen Pract 2010; 60: 489-494.

13 van Mourik Y, Moons KG, Bertens LC, et al. Triage of frail elderly with reduced exercise tolerance in primary care (TREE). A clustered randomized diagnostic study. BMC Public Health 2012; 12: 385.

14 Quanjer PH, Tammeling GJ, Cotes JE, et al. Lung volumes and forced ventilatory flows. Eur Respir J, 1993: 6, Suppl. $16,5 s-40 s$.

15 Rabe KF, Hurd S, Anzueto A, et al. Global strategy for the diagnosis, management, and prevention of chronic obstructive pulmonary disease: GOLD executive summary. Am J Respir Crit Care Med 2007; 176: 532-555.

16 Donders AR, van der Heijden GJ, Stijnen T, et al. Review: a gentle introduction to imputation of missing values. J Clin Epidemiol 2006; 59: 1087-1091.

17 Hurst JR, Vestbo J, Anzueto A, et al. Susceptibility to exacerbation in chronic obstructive pulmonary disease. N Engl J Med 2010; 363: 1128-1138.

18 Wan ES, Demeo DL, Hersh CP, et al. Clinical predictors of frequent exacerbations in subjects with severe chronic obstructive pulmonary disease (COPD). Respir Med 2011; 105: 588-594.

19 Rutten FH, Heddema WS, Daggelders GJ, et al. Primary care patients with heart failure in the last year of their life. Fam Pract 2012; 29: 36-42.

20 Burge S, Wedzicha JA. COPD exacerbations: definitions and classifications. Eur Respir J, 2003: 21, Suppl. 41, 46s-53s.

21 Dimopoulou I, Tsintzas OK, Daganou M, et al. Contribution of lung function to exercise capacity in patients with chronic heart failure. Respiration 1999; 66: 144-149.

22 Faggiano P. Abnormalities of pulmonary function in congestive heart failure. Int J Cardiol 1994; 44: 1-8.

23 Guder G, Rutten FH, Brenner S, et al. The impact of heart failure on the classification of COPD severity. J Card Fail 2012; 18: 637-644.

24 Guerra S, Sherrill DL, Venker C, et al. Morbidity and mortality associated with the restrictive spirometric pattern: a longitudinal study. Thorax 2010; 65: 499-504.

25 Scarlata S, Pedone C, Fimognari FL, et al. Restrictive pulmonary dysfunction at spirometry and mortality in the elderly. Respir Med 2008; 102: 1349-1354.

26 Scarlata S, Costanzo L, Giua R, et al. Diagnosis and prognostic value of restrictive ventilatory disorders in the elderly: a systematic review of the literature. Exp Gerontol 2012; 47: 281-289. 